\title{
Eyebrow duplication-syndactyly syndrome
}

INSERM

\section{Source}

INSERM. (1999). Orphanet: an online rare disease and orphan drug data base. Eyebrow duplication-syndactyly syndrome. ORPHA:3172

Eyebrow duplication-syndactyly syndrome is characterised by partial duplication of the eyebrows and syndactyly of the fingers and toes. It has been described in three patients (a brother and sister and an isolated case). Skin hyperelasticity, hypertrichosis and long eyelashes, and abnormal periorbital wrinkling were also reported in some of the patients. Transmission is autosomal recessive. 